\title{
Cannabidiol for the Treatment of Drug-Resistant Epilepsy in Children: Current State of Research
}

\author{
Christopher L. Anderson ${ }^{1} \quad$ Victoria F. Evans ${ }^{2} \quad$ Thomas B. DeMarse $^{3}$ Marcelo Febo ${ }^{4}$ Cynthia R. Johnson ${ }^{2}$ \\ Paul R. Carney ${ }^{3}$
}

1 Department of Biomedical Engineering, University of Florida, Gainesville, Florida, United States

2 Department of Clinical and Health Psychology, University of Florida, Gainesville, Florida, United States

${ }^{3}$ Department of Neurology, University of North Carolina, Chapel Hill, North Carolina, United States

${ }^{4}$ Department of Psychiatry, University of Florida, Gainesville, Florida, United States

J Pediatr Neurol 2017;15:143-150.

\begin{abstract}
Address for correspondence Christopher L. Anderson, MSc, 1 Hume Area Office, University of Florida, Gainesville, FL 32612, United States (e-mail: Christopher.Anderson@peds.ufl.edu).
\end{abstract}

\author{
Abstract \\ Keywords \\ - cannabidiol \\ - refractory epilepsy \\ - drug-resistant \\ seizures \\ - cannabis \\ - epilepsy
}

The reported effectiveness of these home preparations, especially those with high cannabidiol (CBD) concentrations, has garnered the attention of the medical community. In particular cannabis sativa, known for its lack of a psychoactive effect and high CBD content, has become a target of medical research. The shift in public and political interest to medicinal applications of CBD demands renewed research into its efficacy. Pediatric populations in particular stand to benefit significantly from a better understanding of the safety and efficacy of this novel treatment. This review discusses the current state of $C B D$ research and identifies areas that require further investigation as they pertain to pediatric epilepsy populations. It will especially cover those suffering from refractory epilepsies for which other methods of remediation have not sufficed.

\section{Introduction}

Antiepileptic drugs (AEDs) are the leading treatment option for symptomatic management of seizures for childhood epilepsy. Pharmacologic treatment of epileptic seizure activity has been widely used since the early 1900s with the development of drugs such as phenobarbital, phenytoin, carbamazepine, and valproic acid, all of which are still prescribed by neurologists to this day. ${ }^{1}$ AEDs are not an effective treatment option for all epilepsy diagnoses, however. It is estimated that nearly one-third of all patients with epilepsy are likely to not respond to AED therapies. ${ }^{2}$ When these patients fail to have control of their seizures after an adequate dosage of at least two AEDs, they are categorized as having "drug-resistant" or "refractory" epilepsy. This severe form of epilepsy has a common onset in early childhood and an incidence of around 1 in 40 to $50,000,{ }^{3,4}$ accounting for $4.3 \%$ of all childhood epilepsies. ${ }^{5}$

Among children with drug-resistant epilepsy, those suffering from early-onset and high seizure burden epilepsies suffer the greatest neurodevelopmental problems. Because of the broad spectrum of behavioral symptoms in this population, it is often challenging to identify effective treatments. An optimal medication would improve social interactions and reduce seizure frequency without significant motor, emotional, and cognitive side effects. Recent evidence suggests that more effective early control of epilepsy is associated with better developmental outcomes than in children who were treated 20 to 30 years prior. ${ }^{6}$ As such, an unreported number of families have turned to alternative forms of treatment in an attempt to gain control of their children's health from ineffective AEDs. The most striking of these home remedies is cannabis. Although illegal in many received

September 28, 2016

accepted after revision

December 20, 2016

published online

January 23, 2017
Copyright (c) 2017 by Georg Thieme Verlag KG, Stuttgart · New York
DOI https://doi.org/ 10.1055/s-0037-1598109. ISSN 1304-2580. 
states, many families risk the consequences based on anecdotal evidence of success.

The reported effectiveness of these home preparations, especially those with high cannabidiol (CBD) concentrations has garnered the attention of the medical community and become a target of medical research. The shift in public and political interest to medicinal applications of CBD demands renewed research into its efficacy. Pediatric populations in particular stand to benefit significantly from a better understanding of the safety and efficacy of this novel treatment. This review discusses the current state of CBD research and identifies areas that require further investigation as they pertain to pediatric epilepsy populations. It will especially cover those suffering from refractory epilepsies for which other methods of remediation have not sufficed.

\section{History of Cannabis in Medicine}

Cannabis, one of the world's oldest crops to be cultivated, has been a historically significant part of humankind's way of life, from religion and agriculture to politics and medicine, with its extensive use known to be more than 12,000 years old (Abel) and continuing on to present day. The Ancient Chinese noted that cannabis worked well as an anesthetic for surgeries. ${ }^{7}$ The first documented use of cannabis for treating seizures, which is the focus of our research, occurred was around 1100 AD by Arabic writer alMayusi. ${ }^{8}$ Roman, Germanic, and other European peoples still found significant medicinal value in the plant, using it for relieving pain caused by trauma, in dentistry, and even during childbirth. ${ }^{9,10}$ During the 15 th century historian Ibn al-Badri wrote of an epileptic child being treated with cannabis and "it cured him completely, but he became an addict who could not for a moment be without the drug." 11

Increased world trade continued to move cannabis around the world, finding its way to Columbia, Portugal, Brazil, Chile, and the New World by European influence. Hemp became a staple in the new colonies, where the drug continued to be grown by most farmers, and even required by some townships, to be used to make paper, fishing nets, rope, clothing, and other materials. In the 19th century physicians administered cannabis for patients with epilepsy, including Queen Victoria's personal physician who claimed that "attacks or violent convulsions...may be stopped with a full dose of hemp."12 It was in the 1830 s when the notorious physician William Brooke O'Shaughnessy performed experiments with cannabis and found that it did not have any negative medicinal effects, a major breakthrough that helped dispel some of the damaging beliefs that detracted from some of the benefits the drug could have. Still, cannabis continued to be known more for its nonmedicinal uses, and it became closely associated with alcohol, debt, and poverty when many slaves and plantation workers had continued its recreational use. Evidence and use of the plants medical benefits slipped away from the interest of society, eventually ending in the middle of the prohibition movements of the 1920s in the
United States and the United Kingdom, which included alcohol and other drugs, and where the possession, raising, and consumption of the plant became illegal.

In 1937 the Marijuana Tax Act was passed by Congress, which gave the Drug Enforcement Agency complete control to regulate the cannabis, essentially criminalizing possession of marijuana across the country. ${ }^{13}$ Little changed over the next several decades, though hemp and marijuana did make resurgence in recreational use in the 1960s. The resurgence sparked some interest in its potential uses, but political limitations and conservative views kept any advancement in the medical field nearly nonexistent. Cunha et al conducted clinical trials on healthy volunteers from 1972 to 1980 and published results showing that acute and chronic administration of CBD did not induce psychological or physical symptoms expected from drug toxicity, with the exception of somnolence, nor did the laboratory tests reveal any concerning results. ${ }^{14}$ Today, marijuana is considered a Schedule I controlled substance, along with heroin and lysergic acid diethylamide (LSD), which would indicate that there is no evidence for acceptable, medical, or safe use. Though heavily restricted and fighting an uphill battle against stigma, the Department of Health and Human Services estimates that more than $6 \%$ of the United States population still uses the drug. ${ }^{15}$ The 21 st century has brought with it another wave of progress and views, as a liberal youth movement has led to a majority of people in the United States supporting the legalization of marijuana. ${ }^{16}$

Much of the research looking at marijuana as a treatment option is quite limited, and most knowledge comes from anecdotal reports or preclinical studies on animals. ${ }^{17}$ That being said, there are several reports available, with several case studies and surveys performed over the last few decades showing that cannabis, in various forms, has an anticonvulsant effect on individuals with epilepsy and seizure activity, with fewer and less severe side effects when compared with other Food and Drug Administration (FDA)-approved medications. ${ }^{18}$ Physicians are acknowledging the potential benefits the drug possesses, looking for more human clinical trials to be performed to draw more concise conclusions. As patients seek better treatment options, medical marijuana has become increasingly popular on political and medicinal agendas, with 23 states taking actions to legalize its use for various diseases and ailments. Both the American Medical Association and American College of Physicians are calling for more research to be done as well. ${ }^{19}$

Looking at the long and complicated history of cannabis, it is easy to see how a drug with evidence of strong medical potential was lost in a world controlled by religious and political ideologies. Centuries of various uses and negative connotations have often swayed public opinion and buried the signs of medicinal possibilities, restricting care options to those who are in desperate need of better treatment and quality of life. However, with a new era of compassionate medicine beginning, and a strong movement for legalization brought on by public opinion, cannabis will at last get the attention it desperately needs and deserves from scientists and researchers alike. 


\section{Pharmacologic Properties of Cannabinoid}

As has been detailed, cannabis has shown its potential for the therapeutic treatment of a variety of ailments and diseases. The naturally grown plant contains more than 80 different biochemical compounds called cannabinoids, many of which share very similar chemical structures and active properties. ${ }^{11}$ The two most commonly noted and studied of these composites are delta-9-tetrahydrocannabinol (THC), known for its psychoactive qualities that create an effect many describe as being "high," and CBD, which is the motivating cannabinoid being focused on in this dissertation. CBD is nonpsychotropic, often making it the ideal focus for medicinal research. ${ }^{20}$ The numerous other cannabinoids found in the plant are less in ratio and under studied compared with THC and CBD, though potential medicinal qualities have been shown in some experiments where cannabidivarin (CBDV) and cannabichromene (CBC) may be used as anti-inflammatories or anticonvulsants. ${ }^{21,22}$ Over the years, as legal restrictions have loosened their grip, cannabis growers have been able to develop refined breeding practices for producing plants with both high THC and CBD content. However, this horticulture process is not perfect. Various preferences in techniques and growing capacities of different nurseries lead to variability among plant content, resulting in inconsistent medicinal products for patients. In many cases, the product lacks the pharmaceutical quality control and refinement that other AEDs are subjected to. This is another hurdle making medical research with THC and CBD difficult, as there are several strains and products available to patients with varying cannabinoid content, making consistent measures of efficacy challenging to quantify. Therefore, one particular strain of medical marijuana with a focused cannabinoid and established chemical makeup is very important to draw concise conclusions.

Cannabinoids are often classified into three subgroups: Phytocannabinoids, endocannabinoids, and synthetic cannabinoids. Phytocannabinoids are the cannabinoids that are found naturally in the cannabis plant, of which THC and CBD are the most prevalent. Endocannabinoids are chemical compounds found throughout the central and peripheral nervous systems, which activate receptors for cannabinoids and act as neuromodulators for several neurologic processes. Allyn Howlett and William Devane first discovered and characterized these cannabinoid receptors in a rat brain in $1988 .^{23}$ They found that these receptors were some of the most prevalent in the brain and are far more abundant than any other neurotransmitter receptor, making them a critical component to neurologic processes. Anandamide and 2arachindonoylglycerol (2-AG) are two of the most abundant endocannabinoids in the body and have been shown to regulate immune system functions as well as sensory receptors associated with pain and hunger. ${ }^{24}$ Synthetic cannabinoids are human-made compounds that are vastly different from their natural counterparts but claim to have the psychoactive and sometimes medicinal effects of cannabis. Recent analyses of these synthetic products have found them to be much less effective and have a higher risk of adverse effects including psychotic episodes and seizures. Therefore, medical research is turning away from synthetic cannabinoids and focusing on phytocannabinoids and the effect they may be having within the endocannabinoid system.

\section{Mechanisms of Action of Cannabinoids}

The precise mechanisms of action of cannabinoids are not completely understood, though studies have isolated and characterized both THC and CBD to better recognize their pharmacology. Recent progress in understanding the endogenous cannabinoid system has allowed new insights into the effects of cannabinoids in their anticonvulsive activities. The endocannabinoid system is a very complex physiologic system in which cannabinoid receptors are found to interact with these natural chemicals. When excessive neuronal activity occurs, endocannabinoids are generated on demand and activate G-protein-coupled receptors, known as cannabinoid type 1 (CB1) and type 2 (CB2) receptors. ${ }^{25} \mathrm{CB} 1$ receptors are most prevalently found in the presynaptic region the axon terminal, and regulate both $\gamma$-aminobutyric acid (GABA) and glutamate release from the inhibitory regions. ${ }^{26}$ In a model of epileptiform seizures, it has been shown that $\mathrm{CB} 1$ receptors expressed on excitatory glutamatergic neurons modulate effects in the central nervous system (CNS) and in turn mediate the anticonvulsive activity of endocannabinoids. ${ }^{25,27}$ CB2 receptors are more commonly found on immune and neoplastic cells, and it is expected that they also play a role in neural modulation..$^{25}$ These qualities make the endocannabinoid system and its receptors very important for controlling the rapid firing of neurons that overwhelm the brain and cause seizure activity.

THC has been found to bind to both the CB1 and CB2 receptors and be an agonist. ${ }^{23}$ When excessive neuronal activity occurs, endocannabinoids are generated on demand and activate CB1 receptors. The systemic activation of CB1 receptors by exogenous cannabinoids, such as THC, generally reduces the release of neurotransmitters and in turn reduces or controls activity. However, the application of CB1 agonists does not always target the appropriate neuronal subpopulation containing CB1 receptors and induce a protective effect. ${ }^{27}$ In fact, the opposite may happen, and the treatment could potentially worsen the seizure frequency. This is where $\mathrm{CBD}$, which is understood to be an antagonist for $\mathrm{CB} 1$ and $\mathrm{CB} 2$ receptors, ${ }^{21}$ can be beneficial. Unlike THC, CBD only weakly binds to $\mathrm{CB} 1$ and $\mathrm{CB} 2$ receptors and establishes antiepileptic contrivances likely outside of the endocannabinoid system. ${ }^{28}$ While the exact mechanisms of CBD are not well understood, it is believed that this cannabinoid prevents neuronal hyperexcitability by modulating activity of the neurons via its pharmacologic profile. ${ }^{29} \mathrm{CBD}$ inhibits the CYP2C19 metabolic receptors, which are used by several other AEDs to enter the central nervous system and neuronal pathways. ${ }^{30}$

Some studies have linked CBD to increased inhibition of GABA receptors and regulation of calcium channels, which could also result in an anticonvulsant effect. ${ }^{21}$ Underlying neural mechanisms of epilepsy are often related to increased glutamate/GABA activity, which sometimes results in 
excitotoxicity, or overactivity, that can cause eccentric neuronal connectivity with resulting seizure and behavior deficit. $^{31} \mathrm{CBD}$ controlling glutamate or GABA activity results in decreased epileptic activity as well as having a corresponding change in cognition or behavior. Other noted mechanisms of action of CBD that include antagonizing G-protein-coupled receptors by binding to members of the TRP cation channels at low levels, leading to decreased presynaptic release of glutamate, ${ }^{32,33}$ inhibiting adenosine reuptake, ${ }^{34}$ and activating 5-hydroxytryptophan $1 \mathrm{~A}$ receptors. ${ }^{35} \mathrm{CBD}$ has also been noted to have both antioxidant and anti-inflammatory effects, ${ }^{36}$ leading researchers to believe that it is likely the stacking of all of these effects from CBD that helps modulate neuronal activity and reduce epileptic discharge.

Introducing CBD as a treatment for seizure activity and for patients suffering from intractable generalized epilepsy, where other AEDs are underperforming, could prove to be beneficial, especially for children whose brains are still developing and highly affected by abnormal activity. Recording changes in seizure activity is only one method for quantifying CBD's effectiveness as an antiepileptic treatment option, with behavioral changes and measures of quality of life are also significant to better understand how beneficial this drug can truly be. It is here, as we look to better utilize these pharmacologic properties of cannabis, which we focus on first studying the safety and efficacy of CBD for children with intractable generalized epilepsy. By performing these initial studies, we can start assessing CBD as a treatment option compared with other AEDs and with it begin asking more specific questions on how to make the drug more effective for those patients desperately in need.

\section{Evidence of Cannabis for Childhood Epilepsy and Seizures}

As noted, economic and political interests in the United States have made research scarce, and finding detailed information on the efficacy of the drug as a medicine is a difficult undertaking. Though a majority of this research is anecdotal, there have been several recently published case studies, surveys, and small trials looking at the effects that cannabis has on seizure activity and epilepsy in children. Among children with epilepsy, those suffering from early-onset and high seizures suffer the greatest neurodevelopmental problems, including intellectual disability and behavioral setbacks. $^{37}$ Because of the broad spectrum of behavioral symptoms in this population, it is often challenging to identify effective treatments. An optimal medication would improve social interactions and reduce seizure frequency without significant motor, emotional, and cognitive side effects. An unreported number of families have turned to alternative forms of treatment in an attempt to wrest control of their children's health from ineffective AEDs, with the most common of these home remedies being cannabis. Although still restricted in many states, many families risk the consequences based on anecdotal evidence of success.

In August 2013, CBD became nearly a household name when a CNN news article told its readers of the journey of a young 6-year-old girl named Charlotte Figi, who was diagnosed with Dravet's syndrome, a rare form of epilepsy. ${ }^{38}$ By the age of 3 , Charlotte was having an average of 300 grand mal seizures (the most severe seizure type) per week and her condition was worsening to the point that physicians were suggesting a medically induced coma to give her battered body a rest. Numerous failed treatment attempts and the reality that there was nothing the hospital could do, Charlotte's parents decided to try a specific strain of marijuana for their daughter. The results were immediate, making Charlotte seizure free after a week of use. The larger scientific community immediately started discussing clinical implications of using CBD to treat epilepsy, with many pharmaceutical companies beginning their own drug development and testing. CBD, being a nonpsychoactive, became the ideal focus of interest for children in particular, due to fewer side effects. Significant examination of CBD for human use quickly began, leading to more critical and sophisticated surveys and studies.

Doubled-blind studies using CBD and a placebo control for epilepsy treatment are few, and of those, the low number of patients involved makes it difficult to provide consistent and sufficient data to prove effectiveness of the drug. Though the obvious conclusion of these smaller studies would be to perform them with a larger patient population and a more long-term treatment plan to better assess safety and efficacy, they give us a snapshot of the possible placebo effect that many federal agencies claim could be a possible explanation for "claimed success" with CBD treatment. One of these studies included 15 patients with generalized epilepsy who were randomly divided into two groups, all of which received a double-blind 200 to $300 \mathrm{mg} / \mathrm{kg}$ daily dosage of CBD or placebo along with their current AEDs. ${ }^{14}$ Of the eight who ended up receiving $C B D$, four were seizure free, three had greater than $50 \%$ reduction, and one had no change in activity. Only one of the seven patients in the placebo group noted an improvement in seizure frequency at the end of the trial. Though low in sample size, studies such as this show the importance of using double-blind and placebo controlled studies for CBD research.

One online survey was completed by parents who had administered high-CBD preparations for their children with epilepsy, most of whom were specifically diagnosed with Lennox-Gastaut syndrome. Nearly $85 \%$ of all parents reported that the CBD treatment caused a reduction in their child's seizure frequency, with $14 \%$ claiming they were seizure free. Parents also noted an improvement in daily mood and sleep after taking the drug. ${ }^{39}$ A similar, more comprehensive survey was performed at Stanford University, where 19 parents were surveyed to determine their observations about the effect or lack of effect of CBD on their children's seizure frequency. ${ }^{40}$ The children ranged in age from 2 to 16 years. Thirteen children had Dravet's syndrome (one of whom had epilepsy in female with mental retardation, EMFR), three children had Doose's syndrome, and one each had myoclonic astatic epilepsy, Lennox-Gastaut syndrome, and idiopathic early-onset epilepsy. The children experienced a variety of seizure types, including focal, tonic-clonic, myoclonic, atonic, and infantile spasms, and had unsuccessfully exasperated an 
average of 12 other AEDs before their parents began CBDenriched cannabis treatment. The doses of CBD the parents reported providing ranged from less than $0.5 \mathrm{mg} / \mathrm{kg} / \mathrm{d}$ to 28.6 $\mathrm{mg} / \mathrm{kg} / \mathrm{d}$. The doses of THC contained within those samples were reported to range from 0 to $0.8 \mathrm{mg} / \mathrm{kg} / \mathrm{d}$. To obtain dosage information, parents had their preparations tested at commercial medical cannabis testing facilities. Seizure frequency before administering CBD-enriched cannabis ranged from 2 per week to 250 per day. To validate the survey, the researchers used the same survey on a similar group of parents to enquire about the effects of an AED approved in Europe for Dravet's syndrome, stiripentol (STP).

Sixteen (84\%) of the 19 parents reported a reduction in their child's seizure frequency. Two parents reported that their child became seizure free after more than 4 months of CBD-enriched cannabis use. Of the remaining 14 parents reporting a change in seizure frequency, 8 reported a greater than $80 \%$ reduction in seizure frequency, 3 reported a greater than $50 \%$ seizure frequency reduction, and 3 reported a greater than $25 \%$ seizure frequency reduction. Three parents reported no change. Twelve parents weaned their child from another AED after starting CBD-enriched cannabis treatment. Beneficial effects of CBD-enriched cannabis other than reduced seizures included better mood (15/19, 79\%), increased alertness (14/19, 74\%), better sleep (13/19,68\%), and decreased self-stimulation (6/19, $32 \%)$. Negative side effects included drowsiness (7/19,37\%) and fatigue $(3 / 19,16 \%)$. Limitations of this data include lack of control data, lack of randomization, and blinding associated with open-label use, as well as uncertainty of the dosage in artisanal preparations. There is also inherent error in parent reporting and validity of qualitative data. However, this survey lends hope that CBD may be a useful pharmaceutical alternative to existing therapies in drug-resistant epilepsy, and sheds light on what doses are being used experimentally that appear to be well tolerated.

A more recent study performed by Devinsky et al used an expanded access program to determine the efficacy and safety of CBD (Epidiolex, GW Pharmaceuticals, Salisbury, United Kingdom) in children and young adults with treatment-resistant epilepsy. ${ }^{41}$ Twenty-three patients, ranging from ages 3 to 26 ( 10.4 years old average) entered the study with nine diagnosed with Dravet's syndrome, four had Myoclonic-Absence epilepsy, three Lennox-Gastaut syndrome, and the others with generalized epilepsies. All patients underwent a 4-week baseline period when parents and caregivers recorded all seizures and seizure type in a studyprovided diary. After baseline data were determined, the patients were then given a purified oil-based extract with a known composition of $98 \% \mathrm{CBD}$ at a dose of $5 \mathrm{mg} / \mathrm{kg} / \mathrm{d}$ in addition to their currently used AEDs. Their daily dose was increased by $5 \mathrm{mg} / \mathrm{kg} / \mathrm{d}$ until a maximum dosage of $25 \mathrm{mg} / \mathrm{kg} /$ $\mathrm{d}$ was reached. Patients received the $\mathrm{CBD}$ treatment for a total of 3 months with seizure data continued to be recorded in provided diaries. All patients enrolled completed the 3 months on the study drug, with 9 (39\%) of the 23 patients having a greater than $50 \%$ reduction in seizures and a $32 \%$ median reduction in seizures across all patients. Four (17\%) of the patients were seizure free at the end of the 3 months, with three of the four notably having Dravet's syndrome. It was also noted that of those who entered the study with Dravet's syndrome, $44 \%$ (4/9) had a greater than $50 \%$ reduction in seizures and a $33 \%$ median reduction from baseline, overall. Some notable side effects were also reported, including somnolence $(13 / 23,57 \%)$, fatigue $(13 / 23,57 \%)$, decreased appetite $(5 / 23,22 \%)$, diarrhea $(5 / 23,22 \%)$, and weight loss $(2 / 13,9 \%)$. Because of some of these side effects, the dose of CBD was reduced from 25 to $20 \mathrm{mg} / \mathrm{kg} / \mathrm{d}$, which resulted in improvement of these symptoms. It was also significant that five patients had their clobazam dosage reduced due to its potential interaction with the $\mathrm{CBD}$, causing heavy sedation.

Researchers recently published evidence of CBD use as a treatment of refractory epilepsy in tuberous sclerosis complex (TSC). ${ }^{42}$ Using the same drug, Epidiolex, 10 pediatric patients with a definite diagnosis of TSC and refractory epilepsy were treated for 12 months. The safety, efficacy, and changes in behavior and cognition were assessed throughout the study. The results showed an improvement in response rate over time from 50 to $66 \%$ of the patients having greater than $50 \%$ reduction in seizures after the trial had ended. Parents reported improvements of alertness, verbal communication, cognitive ability, and expression of emotion. It was detailed that more than half of the participants experienced side effects, but most or all were relived after their other AEDs or current CBD does was adjusted. Though this study includes a very small sample size, it suggests that CBD is safe and can be an effective treatment option for children with refractory epilepsy and TSC.

It is also important to note that no current studies report the long-term efficacy and safety of CBD, nor if CBD alters the comorbidities associated with chronic seizures. Most of the studies detailed assess the immediate impact on the individuals being administered CBD and have found the compound to be both safe and with minimal side effects, but they do not reach beyond the short-term study use. Examining the safety of using the drug over time will be necessary, especially when considering its effect on the developing brain of a child. As research continues and the use of THC in conjunction with CBD for medicinal purposes expands, it will be even more crucial to assess the safety of these cannabinoids. Though CBD is sought after for its lack of psychoactive effects, the benefits of THC cannot be ignored. Neither CBD nor THC has ever had any reported deaths associated with overdoses, though related accidents to a high intake have been noted by several news sources. With an increase in emergency room visits in Colorado, which have been correlated to the state-wide legalization of marijuana for recreation and medicinal use, proper research needs to be conducted to either alleviate or caution its use before physicians can appropriately recommend its use to their patients.

These studies demonstrated the benefits of a clinically conducted, case-controlled trial compared with that of survey or observational collected data, with more directly reported data under known and controlled dosages of CBD. We also see that CBD has a significant effect on seizure activity in this young population. With varying epilepsy disorders and limited controls, this study raises many more questions in 
regards to what types of neurologic disorders may have an effect on CBD efficacy, shown by the higher response in Dravet's syndrome patients. GW Pharmaceuticals, the producers of Epidiolex, have openly reported success of their CBD-based drug in children with Dravet's and Lennox-Gastaut syndromes. Moving forward they have begun doubleblind for the same patient populations to address the FDA's concern over placebo controls as well as smaller studies, looking at other epilepsies including the aforementioned TSC study as well as the efficacy in children with generalized refractory epilepsy.

It also suggests that use of CBD may result in other neurologic and behavioral benefits or changes, other than seizure reduction. Scientific evidence and clinical testing is beginning to support the use of CBD as a medicinal therapy, and with it come many more questions about the drug and how it can be studied and used more effectively in medicine. Though parent-reported results can be a valuable measure of efficacy and changes in child behavior, they are susceptible to the placebo effect, making more standard clinical measures of cognition and behavior important in future studies. Performing trials using CBD with a cohort of participants with similar deficits and diseases, as well as control over AED use, will help us better understand the influence of these variables. Simple, yet straightforward studies would assist in demonstrating the causality of the CBD's effects, determining how truly significant this new area of medicine can be while ensuring that the use of other AEDs and treatments are not intervening with the recorded outcomes.

\section{Future Direction of Cannabinoid Testing}

Drug-resistant epilepsy is phenotypically heterogeneous with individuals expressing a range of conditions from mild to severe intellectual disability, sleep disturbances, mood disorders, and psychosis. Within this cohort, more than $25 \%$ of children will have comorbid autism spectrum disorder (ASD). Because of the broad spectrum of behavioral symptoms in this population, it is often challenging to identify effective treatments. An optimal medication would improve social interactions and reduce seizure frequency without significant motor, emotional, and cognitive side effects.

Several lines of evidence support the use of CBD as a broad acting medication that might alleviate seizures while at the same time treat autistic behaviors. CBD alleviates psychosis, anxiety, facilitates REM sleep, and suppresses seizure activity. Therefore, this promising range of therapeutic effects may benefit children with an ASD. To date, there are no clinical studies to determine these effects of $\mathrm{CBD}$ and behavioral problems with face and construct validity. Importantly, clinical imaging studies are needed to establish the effects of CBD on structural and functional connectivity abnormalities that are now recognized to drive the behavioral features of ASD and other neural deficiencies.

While cannabis, and namely CBD, is becoming an increasingly popular topic of both political agendas and research, it is still challenging to find clinical trials being performed. The main reason for this is again tied to the restrictive and legal circumstances surrounding the drug, making it extremely difficult to get any study approved by protective research agencies or even funded due to the inherent unknowns. With marijuana still listed as a Schedule I substance, hurdles are immediately placed in front of any researchers interested in studying the drug in any of its forms. To perform any clinical research with marijuana, you first need a Drug Enforcement Administration (DEA) license, which allows the drug to be legally prescribed as an investigational new drug (IND) and can only be acquired by an experienced physician practicing in the state where the research is allowed. This is commonly a 3- to 4-month process that scrutinizes the applicant's personal proficiencies and records as a clinician. The study then needs to be approved by the Federal Drug Administration (FDA), the National Institute of Health (NIH), and the research-state's Department of Health $(\mathrm{DoH})$, all of which require significant financial backing and time to complete registrations, proving the value of the proposed research.

After those milestones have been reached, the study must meet all standards set forth by the institutional review board (IRB), whose focus is to review, approve, and monitor any biomedical research that is to be performed on human subjects. With very little scientific evidence of CBD use in humans, and especially children, providing enough proof to assist in the risk-benefit analysis of the requested research is extremely difficult. The welfare of the patients who would be participating needs to be protected, and many researchers are still facing the uneducated bias that looms among the community regarding cannabis and its use. Once all reviews are completed and the aforementioned governing bodies give their approval, the process of acquiring the necessary staff, clinical tools and space, study drug, participants who meet study criteria, and maintenance of appropriate protocols begins. Overall, the entire progression of getting a study involving medical marijuana approved, if it is well supported and all of the avenues are not blocked, can take well over 1 year or more to complete.

Scientific evidence and clinical testing are beginning to support the use of CBD as a medicinal therapy, and with it come many more questions about the drug and how it can be studied and used more effectively in medicine. One of the more obvious questions is how much the placebo effect could be playing a role in the reported outcomes of some drug studies. Performing randomized double-blind trials using placebo-controlled subjects would go a long way to answering this initial quandary. By randomly assigning CBD to a cohort of participants with similar deficits and diseases, the influence of unknown variables leading to a possible bias would be eliminated. This study design would assist in demonstrating the causality of the CBD's effects, determining how truly significant this new area of medicine can be while ensuring that the use of other AEDs and treatments are not intervening with the recorded outcomes.

Another insightful technique that avoids patient and guardian placebo effect risk would be to examine patient electroencephalogram (EEG). Examining a patients EEG would help determine if there are any connectivity changes after taking CBD over time. Researchers at the University of Alabama have begun to look at the effect of CBD on short-term 
EEG recordings, finding that there was very little change in EEG background frequency, interictal epileptiform discharges (IEDs), or the number of seizures from before receiving CBD to a maximum dosage of $25 \mathrm{mg} / \mathrm{kg} / \mathrm{d} .{ }^{43,44}$ Though this initial EEG recording did not show any significant changes after receiving $\mathrm{CBD}$, it is important to note that the results of this initial study are restricted by a relatively short EEG recording time (20 minutes) and time on the study drug ( $\sim 1$ month). A longer, more extensive, EEG recording after periods of 3 to 6 months of patients taking CBD would be significant in determining whether there is an effect on overall brain connectivity. Pairing this with behavioral tests and correlating any noted changes in electrical activity of different areas in the brain with functional outcomes would be a major step forward in better understanding the possible effects of CBD.

Though seizure reduction has a significant effect on the patient's quality of life, looking at the other changes and likely improvements that CBD could be causing in social behavior, cognitive function, or motor skills is also important. Improvements or general changes in a patient's lifestyle are important in assessing how effective an AED can be, including CBD. Evaluating these changes while receiving $C B D$ treatment will be an important step in therapeutic testing of the drug. Other clinical trials will need to look at the importance of dosage timing and the effectiveness of CBD in concurrence with other currently prescribed AEDs or any health risks that could be associated with taking these medications together. Examining how the percent of CBD combined with THC or other cannabinoids may work together, how the drug is administered, and at what point in the diseases life are important questions that need to be answered before the true effectiveness of CBD can be measured. With CBD and medical marijuana making a quick entry into medicine and becoming an increasingly significant topic on the political agenda, improved testing techniques, more focused studies on the effectiveness of different forms of $\mathrm{CBD}$, and stronger involvement by clinicians will go a long way in making CBD a more viable option. Combining the promise of this new drug with the sophisticated techniques of clinical trials and laboratory testing can help physicians feel more comfortable about prescribing CBD, and patients about using CBD, while moving this new treatment option forward in the world of medicine.

\section{References}

1 Friedlander WJ. The rise and fall of bromide therapy in epilepsy. Arch Neurol 2000;57(12):1782-1785

2 Begley CE, Famulari M, Annegers JF, et al. The cost of epilepsy in the United States: an estimate from population-based clinical and survey data. Epilepsia 2000;41(3):342-351

3 Hurst DL. Epidemiology of severe myoclonic epilepsy of infancy. Epilepsia 1990;31(4):397-400

4 Yakoub M, Dulac O, Jambaqué I, Chiron C, Plouin P. Early diagnosis of severe myoclonic epilepsy in infancy. Brain Dev 1992;14(5):299-303

5 Trevathan E, Murphy CC, Yeargin-Allsopp M. Prevalence and descriptive epidemiology of Lennox-Gastaut syndrome among Atlanta children. Epilepsia 1997;38(12):1283-1288
6 Chieffo D, Battaglia D, Lettori D, et al. Neuropsychological development in children with Dravet syndrome. Epilepsy Res 2011;95(01/02):86-93

7 Rudgley R. Lost Civilizations of the Stone Age. New York, NY: Free Press; 1998

8 Lozano I. The therapeutic use of cannabis sativa L. in Arabic medicine. Journal of Cannabis Therapeutics 2001;1:63-70

9 Warf B. High points: an historical geography of cannabis. Geogr Rev 2014;104:414-438

10 Bennett C, Osburn L, Osburn J. Green Gold in the Tree of Life: Marijuana in Magic and Religion. Frazier Park, CA: Access Unlimited; 1995

11 Mechoulam R. The Pharmacohistory of Cannabis sativa. Boca Raton, FL: CRC Press; 1986

12 Reynolds JR. Therapeutic uses and toxic effects of cannabis indica. Lancet 1890;1:637-638

13 Schlosser E. Reefer Madness: Sex, Drugs, and Cheap Labor in the American Black Market. Boston, MA: Houghton Mifflin; 2003

14 Cunha JM, Carlini EA, Pereira AE, et al. Chronic administration of cannabidiol to healthy volunteers and epileptic patients. Pharmacology 1980;21(3):175-185

15 Department of Health and Human Services. Results from the 2007 National Survey on Drug Use and Health. Washington, DC: Dept. of Health and Human Services; 2008

16 Pew Research Center. Majority Now Supports Legalizing Marijuana. Washington, DC: U.S. Politics and Policy; 2013

17 Lorenz R. On the application of cannabis in paediatrics and epileptology. Neuroendocrinol Lett 2004;25(1-2):40-44

18 Hill AJ, Williams CM, Whalley BJ, Stephens GJ. Phytocannabinoids as novel therapeutic agents in CNS disorders. Pharmacol Ther 2012;133(1):79-97

19 Hoeffel J. Medical marijuana gets boost from major doctors group [article]. Los Angeles Times. November 11, 2009

20 Borgelt LM, Franson KL, Nussbaum AM, Wang GS. The pharmacologic and clinical effects of medical cannabis. Pharmacotherapy 2013;33(2):195-209

21 Pertwee RG. The diverse CB1 and CB2 receptor pharmacology of three plant cannabinoids: delta9-tetrahydrocannabinol, cannabidiol and delta9-tetrahydrocannabivarin. Br J Pharmacol 2008;153(2):199-215

22 Shinjyo N, Di Marzo V. The effect of cannabichromene on adult neural stem/progenitor cells. Neurochem Int 2013;63(5):432-437

23 Pertwee RG. Cannabinoid pharmacology: the first 66 years. Br J Pharmacol 2006;147(Suppl 1):S163-S171

24 Gonsiorek W, Lunn C, Fan X, Narula S, Lundell D, Hipkin RW. Endocannabinoid 2-arachidonyl glycerol is a full agonist through human type 2 cannabinoid receptor: antagonism by anandamide. Mol Pharmacol 2000;57(5):1045-1050

25 Kano M, Ohno-Shosaku T, Hashimotodani Y, Uchigashima M, Watanabe M. Endocannabinoid-mediated control of synaptic transmission. Physiol Rev 2009;89(1):309-380

26 Katona I, Freund TF. Multiple functions of endocannabinoid signaling in the brain. Annu Rev Neurosci 2012;35:529-558

27 Lutz B. On-demand activation of the endocannabinoid system in the control of neuronal excitability and epileptiform seizures. Biochem Pharmacol 2004;68(9):1691-1698

28 Wallace MJ, Blair RE, Falenski KW, Martin BR, DeLorenzo RJ. The endogenous cannabinoid system regulates seizure frequency and duration in a model of temporal lobe epilepsy. J Pharmacol Exp Ther 2003;307(1):129-137

29 Jones NA, Hill AJ, Smith I, et al. Cannabidiol displays antiepileptiform and antiseizure properties in vitro and in vivo. J Pharmacol Exp Ther 2010;332(2):569-577

30 Geffrey AL, Pollack SF, Bruno PL, Thiele EA. Drug-drug interaction between clobazam and cannabidiol in children with refractory epilepsy. Epilepsia 2015;56(8):1246-1251

31 Bittigau P, Sifringer M, Ikonomidou C. Antiepileptic drugs and apoptosis in the developing brain. Ann N Y Acad Sci 2003; 993:103-114, discussion 123-124

32 De Petrocellis L, Ligresti A, Moriello AS, et al. Effects of cannabinoids and cannabinoid-enriched cannabis extracts on TRP 
channels and endocannabinoid metabolic enzymes. Br J Pharmacol 2011;163(7):1479-1494

33 Sylantyev S, Jensen TP, Ross RA, Rusakov DA. Cannabinoid- and lysophosphatidylinositol-sensitive receptor GPR55 boosts neurotransmitter release at central synapses. Proc Natl Acad Sci U S A 2013;110(13):5193-5198

34 Carrier EJ, Auchampach JA, Hillard CJ. Inhibition of an equilibrative nucleoside transporter by cannabidiol: a mechanism of cannabinoid immunosuppression. Proc Natl Acad Sci U S A 2006;103(20): 7895-7900

35 Campos AC, Ferreira FR, Guimarães FS. Cannabidiol blocks longlasting behavioral consequences of predator threat stress: possible involvement of 5HT1A receptors. J Psychiatr Res 2012;46(11): 1501-1510

36 Hampson AJ, Grimaldi M, Axelrod J, Wink D. Cannabidiol and (-) $\Delta$ 9-tetrahydrocannabinol are neuroprotective antioxidants. Proc Natl Acad Sci U S A 1998;95(14):8268-8273

37 Pack AM. SUDEP: What are the risk factors? Do seizures or antiepileptic drugs contribute to an increased risk?. Epilepsy Curr 2012;12(4):131-132
38 Young S. Marijuana stops child's severe seizures [article]. Cable News Network. August 7, 2013

39 Hussain SA, Zhou R, Jacobson C, et al. Perceived efficacy of cannabidiol-enriched cannabis extracts for treatment of pediatric epilepsy: a potential role for infantile spasms and Lennox-Gastaut syndrome. Epilepsy Behav 2015;47:138-141

40 Porter BE, Jacobson C. Report of a parent survey of cannabidiolenriched cannabis use in pediatric treatment-resistant epilepsy. Epilepsy Behav 2013;29(3):574-577

41 Devinsky O, Sullivan J, Friedman D, et al. Efficacy and safety of Epidiolex (cannabidiol) in children with treatment-resistant epilepsy: initial data from an expanded access program [abstract]. American Epilepsy Society; 2014

42 Hess EJ, Moody KA, Geffrey AL, et al. Cannabidiol as a new treatment for drug-resistant epilepsy in tuberous sclerosis complex. Epilepsia 2016;57(10):1617-1624

43 Perry L, Szaflarski J, Bebin E. Investigating the effect of cannabidiol on the EEG. J Neurol 2016;86:S14.007-S14.007

44 Friedman D, Devinsky O. Cannabinoids in the treatment of epilepsy. N Engl J Med 2015;373(11):1048-1058 